\title{
A System of Nonlinear Variational Inclusions with $(A, \eta)$-Monotone Mappings
}

\author{
Zhiguo Wang ${ }^{1}$ and Changqun $\mathrm{Wu}^{2}$ \\ ${ }^{1}$ School of Mathematics and Information Sciences, Henan University, Kaifeng 475001, China \\ ${ }^{2}$ School of Business and Administration, Henan University, Kaifeng 475001, China \\ Correspondence should be addressed to Zhiguo Wang, henumath@yahoo.cn
}

Received 17 December 2007; Accepted 2 January 2008

Recommended by Ram U. Verma

A new system of nonlinear variational inclusions involving $(A, \eta)$-monotone mappings in the framework of Hilbert space is introduced and then based on the generalized resolvent operator technique associated with $(A, \eta)$-monotonicity, the approximation solvability of solutions using an iterative algorithm is investigated. Since $(A, \eta)$-monotonicity generalizes $A$-monotonicity and $H$ monotonicity, our results improve and extend the recent ones announced by many others.

Copyright (C) 2008 Z. Wang and C. Wu. This is an open access article distributed under the Creative Commons Attribution License, which permits unrestricted use, distribution, and reproduction in any medium, provided the original work is properly cited.

\section{Introduction}

Variational inclusions problems are among the most interesting and intensively studied classes of mathematical problems and have wide applications in the fields of optimization and control, economics and transportation equilibrium, and engineering sciences. Variational inclusions problems have been generalized and extended in different directions using the novel and innovative techniques. Various kinds of iterative algorithms to solve the variational inequalities and variational inclusions have been developed by many authors. For details, we can refer to [1-9]. In most of the resolvent operator methods, the maximal monotonicity has played a key role, but more recently introduced notions of $A$-monotonicity [7] and $H$-monotonicity [2, 3] have not only generalized the maximal monotonicity, but gave a new edge to resolvent operator methods. Recently, Verma [9] generalized the recently introduced and studied notion of $A$-monotonicity to the case of $(A, \eta)$-monotonicity, while examining the sensitivity analysis for a class of nonlinear variational inclusion problems based on the generalized resolvent operator technique. Resolvent operator techniques have been in use for a while in literature, especially with the general framework involving set-valued maximal monotone mappings, but it got a new empowerment by the recent developments of $A$-monotonicity and $H$-monotonicity. Inspired and motivated by the recent research going on in this area, we introduce and analyze 
a new class of variational inclusions problems involving $(A, \eta)$-monotone mappings [9] in the framework of Hilbert space. Since $(A, \eta)$-monotonicity generalizes $A$-monotonicity [7] and $H$-monotonicity $[2,3]$, our results improve and extend the recent ones announced by many others.

\section{Preliminaries}

In this section, we explore some basic properties derived from the notion of $(A, \eta)$ monotonicity. Let $H$ denote a real Hilbert space with the norm $\|\cdot\|$ and inner product $\langle\cdot, \cdot\rangle$, respectively. Let $\eta: H \times H: \rightarrow H$ be a single-valued mapping. The map $\eta$ is called $\tau$-Lipschitz continuous if there is a constant $\tau>0$ such that $\|\eta(u, v)\| \leq \tau\|y-v\|$ for all $u, v \in H$.

Let $M: H \rightarrow 2^{H}$ be a multivalued mapping from a Hilbert space $H$ to $2^{H}$, the power set of $H$. We recall the following.

(i) The set $D(M)$, defined by $D(M)=\{u \in H: M(u) \neq \varnothing\}$, is called the effective domain of $M$.

(ii) The set $R(M)$, defined by $R(M)=\bigcup_{u \in H} M(u)$, is called the range of $M$.

(iii) The set $G(M)$, defined by $G(M)=\{(u, v) \in H \times H: u \in D(M), v \in M(u)\}$, is the graph of $M$.

Definition 2.1. Let $\eta: H \times H \rightarrow H$ be a single-valued mapping and let $M: H \rightarrow 2^{H}$ be a multivalued mapping on $H$. The map $M$ is said to be

(i) $(r, \eta)$-strongly monotone if

$$
\left\langle u^{*}-v^{*}, \eta(u, v)\right\rangle \geq r\|u-v\| \quad \forall\left(u, u^{*}\right),\left(v, v^{*}\right) \in G(M),
$$

(ii) $(m, \eta)$-relaxed monotone if there exists a positive constant $m$ such that

$$
\left\langle u^{*}-v^{*}, \eta(u, v)\right\rangle \geq-m\|u-v\|^{2} \quad \forall\left(u, u^{*}\right),\left(v, v^{*}\right) \in G(M) .
$$

Definition 2.2 (see [9]). Let $A: H \rightarrow H$ and $\eta: H \times H \rightarrow H$ be two single-valued mappings. The map $M: H \rightarrow 2^{H}$ is said to be $(A, \eta)$-monotone if

(i) $M$ is $(m, \eta)$-relaxed monotone,

(ii) $R(A+\rho M)=H$ for $\rho>0$.

Note that, alternatively, the map $M: H \rightarrow 2^{H}$ is said to be $(A, \eta)$-monotone if

(i) $M$ is $(m, \eta)$-relaxed monotone,

(ii) $A+\rho M$ is $\eta$-pseudomonotone for $\rho>0$.

Definition 2.3. Let $A: H \rightarrow H$ be an $(r, \eta)$-strong monotone mapping and let $M: H \rightarrow H$ be an $(A, \eta)$-monotone mapping. Then the generalized resolvent operator $J_{M, \rho}^{A, \eta}: H \rightarrow H$ is defined by $J_{M, \rho}^{A, \eta}(u)=(A+\rho M)^{-1}(u)$ for all $u \in H$, where $\rho>0$ is a constant. 
Definition 2.4. The map $N: H \times H$ is said to be relaxed $(\beta, \gamma)$-cocoercive with respect to $A$ in the first argument if there exist two positive constants $\alpha, \beta$ such that $\langle T(x, u)-T(y, u), A x-A y\rangle \geq$ $(-\beta)\|T(x, u)-T(y, u)\|^{2}+\gamma\|x-y\|^{2}$ for all $(x, y, u) \in H \times H \times H$.

Proposition 2.5 (see [9]). Let $\eta: H \times H \rightarrow H$ be a single-valued mapping, let $A: H \rightarrow H(r, \eta)$ strongly monotone mapping, and let $M: H \rightarrow 2^{H}$ be an $(A, \eta)$-monotone mapping. Then the mapping $(A+\rho M)^{-1}$ is single valued.

\section{Results on algorithmic convergence analysis}

Let $N: H \times H \rightarrow H$ and $\eta: H \times H \rightarrow H$ be two nonlinear mappings. Let $M: H \rightarrow 2^{H}$ be an $(A, \eta)$-monotone mapping. Then we have the nonlinear system of variational inclusion (NSVI) problem: determine an element $(u, v) \in H \times H$ such that

$$
\begin{aligned}
& 0 \in A u-A v+\rho_{1}[N(v, u)+M(u)], \\
& 0 \in A v-A u+\rho_{2}[N(u, v)+M(v)] .
\end{aligned}
$$

If $u=v$ and $\rho_{1}=\rho_{2}$ in NSVI (3.1), we have the following NVI problem: find elements $u, v \in H$ such that

$$
0 \in N(u, u)+M(u)
$$

which was considered by Verma [8].

In order to prove our main results, we need the following lemmas.

Lemma 3.1 (see [10]). Assume that $\left\{a_{n}\right\}$ is a sequence of nonnegative real numbers such that $a_{n+1} \leq$ $\left(1-\lambda_{n}\right) a_{n}+b_{n}$ for all $n \geq n_{0}$, where $n_{0}$ is some nonnegative integer, $\left\{\lambda_{n}\right\}$ is a sequence in $(0,1)$ with $\sum_{n=1}^{\infty} \lambda_{n}=\infty, b_{n}=\circ\left(\lambda_{n}\right)$, then $\lim _{n \rightarrow \infty} a_{n}=0$.

Lemma 3.2 (see [9]). Let $H$ be a real Hilbert space and let $\eta: H \times H \rightarrow H$ be a $\tau$-Lipschitz continuous nonlinear mapping. Let $A: H \rightarrow H$ be an $(r, \eta)$-strongly monotone and let $M: H \rightarrow 2^{H}$ be $(A, \eta)$ monotone. Then the generalized resolvent operator $J_{M, \rho}^{A, \eta}: H \rightarrow H$ is $\tau /(r-\rho m)$, that is,

$$
\left\|J_{M, \rho}^{A, \eta}(x)-J_{M, \rho}^{A, \eta}(y)\right\| \leq \frac{\tau}{r-\rho m}\|x-y\| \quad \forall x, y \in H .
$$

Lemma 3.3. Let $H$ be a real Hilbert space, let $A: H \rightarrow H$ be $(r, \eta)$-strongly monotone, and let $M$ : $H \rightarrow 2^{H}$ be $(A, \eta)$-monotone. Let $\eta: H \times H \rightarrow H$ be a $\tau$-Lipschitz continuous nonlinear mapping. Then $(u, v)$ is the solution of NSVI (3.1) if and only if it satisfies

$$
\begin{aligned}
& u=J_{M, \rho_{1}}^{A, \eta}\left[A v-\rho_{1} N(v, u)\right], \\
& v=J_{M, \rho_{2}}^{A, \eta}\left[A u-\rho_{2} N(u, v)\right] .
\end{aligned}
$$

Proof. The fact directly follows from Definition 2.4 .

Algorithm 3.4. For any $u_{0}, v_{0} \in H$, compute the sequences $\left\{u_{n}\right\}$ and $\left\{v_{n}\right\}$ by the iterative process

$$
\begin{aligned}
u_{n+1} & =\left(1-\alpha_{n}\right) u_{n}+\alpha_{n} J_{M, \rho_{1}}^{A, \eta}\left[A v_{n}-\rho_{1} N\left(v_{n}, u_{n}\right)\right], \\
v_{n} & =J_{M, \rho_{2}}^{A, \eta}\left[A u_{n}-\rho_{2} N\left(u_{n}, v_{n}\right)\right] .
\end{aligned}
$$

If $u_{n}=v_{n}$ for all $n$ and $\rho_{1}=\rho_{2}$ in Algorithm 3.4, then we have the following algorithm. 
Algorithm 3.5. For any $u_{0} \in H$, compute the sequence $\left\{u_{n}\right\}$ by the iterative processes:

$$
u_{n+1}=\left(1-\alpha_{n}\right) u_{n}+\alpha_{n} J_{M, \rho}^{A, \eta}\left[A u_{n}-\rho N\left(u_{n}, u_{n}\right)\right]
$$

Theorem 3.6. Let $H$ be a real Hilbert space, let $A: H \times H \rightarrow H$ be $(r, \eta)$-strongly monotone and sLipschitz continuous, and let $M: H \rightarrow 2^{H}$ be $(A, \eta)$-monotone. Let $\eta: H \times H \rightarrow H$ be a $\tau$-Lipschitz continuous nonlinear mapping, let $N: H \times H \rightarrow H$ be relaxed $(\beta, \gamma)$-cocoercive (with respect to $A$ ) and $\mu$-Lipschitz coninuous in the first variable, and let $N_{i}$ be $v$-Lipschitz continuous in the second variable. Let $\left(u^{*}, v^{*}\right)$ be the solution of NSVI problem (3.1), and $\left\{u_{n}\right\}$ and $\left\{v_{n}\right\}$ sequences generated by Algorithm 3.4. Suppose the following conditions are satisfied:

(i) $\alpha_{n} \subset(0,1], \sum_{n=0}^{\infty} \alpha_{n}=\infty$;

(ii) $\tau\left(\theta_{1} \theta_{2} \theta_{3}+\rho_{1} v\right)<r-\rho_{1} m$, where $\theta_{1}=\sqrt{s^{2}-2 \rho_{1} \gamma+2 \rho_{1} \beta \mu^{2}+\rho_{1}^{2} \mu^{2}}, \quad \theta_{2}=$ $\sqrt{s^{2}-2 \rho_{2} \gamma+2 \rho_{2} \beta \mu^{2}+\rho_{2}^{2} \mu^{2}}$, and $\theta_{3}=\tau /\left(r-\rho_{2} m-\tau \rho_{2} v\right)$

(iii) $\rho_{2}<r /(m+\tau \mathcal{v})$.

Then the sequences $\left\{u_{n}\right\}$ and $\left\{v_{n}\right\}$ converge strongly to $u^{*}$ and $v^{*}$, respectively.

Proof. Let $\left(u^{*}, v^{*}\right) \in H$ be the solution of NSVI problem (3.1), we have

$$
\begin{aligned}
& u^{*}=\left(1-\alpha_{n}\right) u^{*}+\alpha_{n} J_{M, \rho_{1}}^{A, \eta}\left[A v^{*}-\rho_{1} N\left(v^{*}, u^{*}\right)\right], \\
& v^{*}=J_{M, \rho_{2}}^{A, \eta}\left[A u^{*}-\rho_{2} N\left(u^{*}, v^{*}\right)\right] .
\end{aligned}
$$

It follows that

$$
\begin{aligned}
& \left\|u_{n+1}-u^{*}\right\| \\
& =\left\|\left(1-\alpha_{n}\right) u_{n}+\alpha_{n} J_{M, \rho_{1}}^{A, \eta}\left[A v_{n}-\rho_{1} N\left(v_{n}, u_{n}\right)\right]-u^{*}\right\| \\
& \leq\left(1-\alpha_{n}\right)\left\|u_{n}-u^{*}\right\|+\frac{\tau \alpha_{n}}{r-\rho_{1} m}\left[\left\|A v_{n}-A v^{*}-\rho_{1}\left[N\left(v_{n}, u_{n}\right)-N\left(v^{*}, u_{n}\right)\right]\right\|+\rho_{1}\left\|N\left(v^{*}, u_{n}\right)-N\left(v^{*}, u^{*}\right)\right\|\right] .
\end{aligned}
$$

It follows from relaxed $(\beta, \gamma)$-cocoercive monotonicity and $\mu$-Lipschitz continuity of $N$ in the first variable that

$$
\begin{aligned}
& \left\|A v_{n}-A v^{*}-\rho_{1}\left(N\left(v_{n}, u_{n}\right)-N\left(v^{*}, u_{n}\right)\right)\right\|^{2} \\
& \quad=\left\|A v_{n}-A v^{*}\right\|^{2}-2 \rho_{1}\left\langle N\left(v_{n}, u_{n}\right)-N\left(v^{*}, u_{n}\right), A v_{n}-A u^{*}\right\rangle+\rho_{1}^{2}\left\|N\left(v_{n}, u_{n}\right)-N\left(v^{*}, u_{n}\right)\right\|^{2} \\
& \quad \leq \theta_{1}^{2}\left\|v_{n}-v^{*}\right\|^{2}
\end{aligned}
$$

where $\theta_{1}=\sqrt{s^{2}-2 \rho_{1} \gamma+2 \rho_{1} \beta \mu^{2}+\rho_{1}^{2} \mu^{2}}$. Observe that the $\mathcal{v}$-Lipschitz continuity of $N$ in the second argument yields that

$$
\left\|N\left(v^{*}, u_{n}\right)-N\left(v^{*}, u^{*}\right)\right\| \leq v\left\|u_{n}-u^{*}\right\| .
$$


On the other hand, we have

$$
\begin{aligned}
\left\|v_{n}-v^{*}\right\| & =\left\|J_{M, \rho_{2}}^{A, \eta}\left[A u_{n}-\rho_{2} N\left(u_{n}, v_{n}\right)\right]-v^{*}\right\| \\
& \leq \frac{\tau}{r-\rho_{2} m}\left\|A u_{n}-A u^{*}-\rho_{2}\left[N\left(u_{n}, v_{n}\right)-N\left(u^{*}, v^{*}\right)\right]\right\| \\
& \leq \frac{\tau}{r-\rho_{2} m}\left[\left\|A u_{n}-A u^{*}-\rho_{2}\left[N\left(u_{n}, v_{n}\right)-N\left(u^{*}, v_{n}\right)\right]\right\|+\rho_{2}\left\|N\left(u^{*}, v_{n}\right)-N\left(u^{*}, v^{*}\right)\right\|\right] .
\end{aligned}
$$

It follows from relaxed $(\beta, \gamma)$-cocoercive monotonicity and $\mu$-Lipschitz continuity of $N$ in the first variable that

$$
\begin{aligned}
& \left\|A u_{n}-A u^{*}-\rho_{2}\left(N\left(u_{n}, v_{n}\right)-N\left(u^{*}, v_{n}\right)\right)\right\|^{2} \\
& \quad=\left\|A u_{n}-A u^{*}\right\|^{2}-2 \rho_{2}\left\langle N\left(u_{n}, v_{n}\right)-N\left(u^{*}, v_{n}\right), A u_{n}-A u^{*}\right\rangle+\rho_{2}^{2}\left\|N\left(u_{n}, v_{n}\right)-N\left(u^{*}, v_{n}\right)\right\|^{2} \\
& \quad \leq \theta_{2}^{2}\left\|u_{n}-u^{*}\right\|^{2}
\end{aligned}
$$

where $\theta_{2}=\sqrt{s^{2}-2 \rho_{2} \gamma+2 \rho_{2} \beta \mu^{2}+\rho_{2}^{2} \mu^{2}}$. Again, observe that the $v$-Lipschitz continuity of $N_{2}$ in the second argument yields that

$$
\left\|N\left(u^{*}, v_{n}\right)-N\left(u^{*}, v^{*}\right)\right\| \leq v\left\|v_{n}-v^{*}\right\| .
$$

Substituting (3.12) and (3.13) into (3.11) yields that

$$
\left\|v_{n}-v^{*}\right\| \leq \frac{\tau}{r-\rho_{2} m}\left[\theta_{2}\left\|u_{n}-u^{*}\right\|+\rho_{2} v\left\|v_{n}-v^{*}\right\|\right]
$$

It follows from condition (iii) that

$$
\left\|v_{n}-v^{*}\right\| \leq \theta_{3} \theta_{2}\left\|u_{n}-u^{*}\right\|
$$

where $\theta_{3}=\tau /\left(r-\rho_{2} m-\tau \rho_{2} v\right)$. Substituting (3.15) into (3.9), we obtain

$$
\left\|A v_{n}-A v^{*}-\rho_{1}\left(N\left(v_{n}, u_{n}\right)-N\left(v^{*}, u_{n}\right)\right)\right\| \leq \theta_{1} \theta_{2} \theta_{3}\left\|u_{n}-u^{*}\right\| .
$$

Again, substituting (3.10) and (3.16) into (3.8), we arrive at

$$
\begin{aligned}
\| u_{n+1} & -u^{*} \| \\
& \leq\left(1-\alpha_{n}\right)\left\|u_{n}-u^{*}\right\|+\frac{\tau \alpha_{n}}{r-\rho_{1} m}\left[\theta_{1} \theta_{2} \theta_{3}\left\|u_{n}-u^{*}\right\|+\rho_{1} v\left\|u_{n}-u^{*}\right\|\right] \\
& =\left[1-\alpha_{n}\left(1-\frac{\tau}{r-\rho_{1} m}\left(\theta_{1} \theta_{2} \theta_{3}+\rho_{1} v\right)\right)\right]\left\|u_{n}-u^{*}\right\| .
\end{aligned}
$$

Using conditions (i)-(iii) and applying Lemma 3.1 to (3.17), we can obtain the desired conclusion. This completes the proof. 
From Theorem 3.6, we have the following result immediately.

Corollary 3.7. Let $H$ be a real Hilbert space, let $A: H \times H$ be $(r, \eta)$-strongly monotone and s-Lipschitz continuous, and let $M: H \rightarrow 2^{H}$ be $(A, \eta)$-monotone. Let $\eta: H \times H \rightarrow H$ be a $\tau$-Lipschitz continuous nonlinear mapping, let $N: H \times H \rightarrow H$ be relaxed $(\beta, \gamma)$-cocoercive (with respect to $A$ ) and $\mu$-Lipschitz coninuous in the first variable, and let $N$ be $v$-Lipschitz continuous in the second variable. Let $u^{*}$ be the solution of NVI problem (3.2), and $\left\{u_{n}\right\}$ a sequence generated by Algorithm 3.5. Suppose the following conditions are satisfied:

(i) $\alpha_{n} \subset(0,1], \sum_{n=0}^{\infty} \alpha_{n}=\infty$;

(ii) $\tau(\theta+\rho v)<r-\rho m$, where $\theta=\sqrt{s^{2}-2 \rho \gamma+2 \rho \beta \mu^{2}+\rho^{2} \mu^{2}}$.

Then the sequence $\left\{u_{n}\right\}$ converges strongly to $u^{*}$.

\section{References}

[1] R. P. Agarwal, Y. J. Cho, and N. J. Huang, "Sensitivity analysis for strongly nonlinear quasi-variational inclusions," Applied Mathematics Letters, vol. 13, no. 6, pp. 19-24, 2000.

[2] Y. P. Fang and N. J. Huang, " $H$-monotone operator and resolvent operator technique for variational inclusions," Applied Mathematics and Computation, vol. 145, no. 2-3, pp. 795-803, 2003.

[3] Y. P. Fang and N. J. Huang, " $H$-monotone operators and system of variational inclusions," Communications on Applied Nonlinear Analysis, vol. 11, no. 1, pp. 93-101, 2004.

[4] Y. P. Fang, N. J. Huang, and H. B. Thompson, "A new system of variational inclusions with $(H, \eta)-$ monotone operators in Hilbert spaces," Computers \& Mathematics with Applications, vol. 49, no. 2-3, pp. 365-374, 2005.

[5] N. J. Huang and Y. P. Fang, "A new class of general variational inclusions involving maximal $\eta$ monotone mappings," Publicationes Mathematicae Debrecen, vol. 62, no. 1-2, pp. 83-98, 2003.

[6] R. U. Verma, "Sensitivity analysis for relaxed cocoercive nonlinear quasivariational inclusions," Journal of Applied Mathematics and Stochastic Analysis, vol. 2006, no. 3, Article ID 52041, 9 pages, 2006.

[7] R. U. Verma, "A-monotonicity and applications to nonlinear variational inclusion problems," Journal of Applied Mathematics and Stochastic Analysis, vol. 2004, no. 2, pp. 193-195, 2004.

[8] R. U. Verma, "A-monotone nonlinear relaxed cocoercive variational inclusions," Central European Journal of Mathematics, vol. 5, no. 2, pp. 386-396, 2007.

[9] R. U. Verma, "Approximation solvability of a class of nonlinear set-valued variational inclusions involving $(A, \eta)$-monotone mappings," Journal of Mathematical Analysis and Applications, vol. 337, no. 2, pp. 969-975, 2008.

[10] X. L. Weng, "Fixed point iteration for local strictly pseudo-contractive mapping," Proceedings of the American Mathematical Society, vol. 113, no. 3, pp. 727-731, 1991. 